\title{
Implementing performance improvement through the enterprise culture
}

\author{
Paul Turner, Ros Kane, Christine Jackson
}

\section{ABSTRACT}

The purpose of this article is to evaluate performance improvements made through a case study of a rural English hospital's emergency department within the framework of the enterprise culture-the NHS adoption of the private sector's performance improvement best practice. Additionally, the process of the research and the significant barriers encountered are documented to inform future researchers of potential drawbacks when considering this type of research. An intervention addressing local performance issues was defined by medical consultants. A pathway to care for emergency care patients requiring clinical observation for more than four hours-but not admission as a hospital inpatient-was created. The efficacy of the intervention was evaluated by measuring compliance to the pathway standards and conducting an ethnographic study and semi-structured interviews. Structural barriers to research caused by lack of staff availability were encountered. Additionally, although improvement from the intervention was encouraging, the ability of the people within the system to consistently enact the process caused performance issues against expected targets.

Key Words: Emergency department • Innovation • Barriers to research • NHS change

This is the seventh article in a series recording the study undertaken by the principal author. The first four articles examined the development of policy leading to the enterprise culture, which looks to make quality and efficiency improvements in the English NHS (Wall and Owen, 2003: 113-5). The Purpose, Process, People (PPP) framework (Womack, 2011) used in private sector best practice, which underpins the enterprise culture, is also examined.

The fifth and sixth articles tested a deductive theory challenging whether the enterprise culture has provided a framework for performance improvement in a case study at a single emergency department in a rural district general hospital (DGH) (Turner et al, 2014). The fifth article analysed the purpose element of the PPP framework and concludes that the data describing patient demand can be analysed to provide work rates to attain the four-hour wait target using PPP techniques (George, 2003; Miltenburg, 2007). The sixth article addressed the process and people elements to evaluate the capacity characteristics of the enterprise culture within the emergency department and their effects on performance against the four-hour wait target. This article supports Silvester et al's (2004) findings that emergency care capacity
Paul Turner

Senior Lecturer, University of Lincoln

Ros Kane Principal Lecturer, University of Lincoln

Christine Jackson Principal Research Fellow, University of Lincoln

Email:Paul.turner@ulh. nhs.uk 
is not planned to meet demand characteristics. The article concludes that the four-hour wait target performance is frequently a product of reactionary management intervention and not care standards or processes (Turner et al, 2015c)

This article aims to test the hypothesis that enterprise culture framework present in the emergency department is successful in achieving improvement in line with its quality and efficiency aims.

\section{Methods}

To test the hypothesis, an intervention was created by the two substantive medical consultants at the emergency department. To expose the efficacy of the enterprise culture present within the emergency department, the consultants chose an important performance issue within their area of control and designed an intervention to improve it. To support the choice of performance issues, the validated findings of the capacity, demand and performance analysis were presented to the consultants for reference, but they were at liberty to select their own departmental need for improvement.

Validation of the capacity, demand and performance data from participants within the system provides critique of the findings and was chosen because they 'have access to additional knowledge of the context [of the system] ... that is not available to the ethnographer' (Hammersley and Atkinson, 1995: 228). A series of modified Delphi studies were planned to validate the capacity and demand and performance analysis. Participants were asked the extent to which they agreed that that the analyses were an accurate description of operational activities within the emergency department. A nine-point Likert scale and a median score of seven were used to achieve consensus. Findings from the capacity, demand and performance studies are discussed in previous articles (Turner et al, 2015b; 2015c).

\section{Methods to define and evaluate the intervention}

The purpose of the intervention was to enable the emergency department to provide a solution to a locally identified problem under the established enterprise culture. The efficacy of the intervention was then evaluated quantitatively and through further ethnographic observation and semi-structured interviews.

To measure the effect of the intervention, sets of patient notes were reviewed by the clinical director. In total, 17 sets of notes from patients following the intervention were randomly selected from the week following implementation of the intervention. The sample size reflected only the time that the clinical director was able to commit and not a number calculated to give precision to a statistical test. As the intervention chosen by the consultants represented a new process, comparative tests against previous performance were not possible. However, the proportion of patients complying with the intervention indicators (when reviewed by the clinical director) was calculated. The confidence level of the sample proportion was calculated to define a range of values in which population performance is likely to lie (Campbell et al, 2007: 89).

The intervention and its target and process were updated in the staff handbook and communicated by disseminated staff briefings and email by the clinical director.

\section{The capacity,demand and performance analysis}

An open question Delphi round to 'obtain ideas or attitudes' (Bowling, 2009:437) about capacity, demand and performance influences, was undertaken using online survey software. The research findings and summarised comments from the Delphi round, was then presented to a smaller nominal group to achieve consensus. No new knowledge was added from the open round and validation was achieved by full consensus from the participants. This approach was preferred to several interviews with individuals because groups provide greater 'access to interaction between participants, and thus some insight into how social knowledge is produced' (Green and Thorogood, 2004: 107). It is noted that groups may be open to participants exerting more influence however, given the small number of participants available from the emergency department staff, we considered the benefits of interaction more compelling. Bloor et al suggest that a minimum Delphi group size is 
four, however, they argue that a more important factor in the group dynamic is a balance of 'viewpoints, experience and interests' (2015: 66) and the study aimed to achieve this within the restrictions of the limited number of potential participants from a small, rural DGH.

\section{Intervention}

Treatment of a cohort of emergency care patients requiring clinical observation for at least 12 hours after attendance was chosen by the consultants to address performance issues through the intervention. Observation patients were defined as those who had presented to, and been assessed and treated in, the emergency department. Although they did not require admission to an acute bed, these patients were kept under observation for a limited time. The treatment of observation patients was chosen because it represented a critical local need which was caused by a gap in clinical control (namely a lack of agreed process) within the emergency department and the clinical decision unit (CDU). Observation and admitted medical patients were transferred to the CDU prior to their discharge or relocation to a specialty ward.

The indicators assigned by the consultants to measure success in the intervention were:

- Whether the observation patient had a care plan agreed and documented by an emergency department doctor or nurse

- Whether a drug chart was complete when the patient was transferred to CDU.

As these indicators were essential elements of clinical care, complete compliance was expected.

From the 17 sets of notes reviewed, 15 patients (a proportion of 0.882 ) had a clinically appropriate plan and drug chart. However, this infers with $95 \%$ confidence that performance fell between $63.5 \%$ and $98.5 \%$ of the population of patients using the pathway during that week.

\section{Evaluating the intervention}

The confidence interval carried risks when

drawing conclusions about the efficacy of the intervention, namely: the limitations of the sample size and the possibilities of the results being unrepresentative of long-term performance. This led to a need to triangulate the quantitative analysis of the intervention. Two methods were devised to achieve this-a further period of ethnographic observation and interviews with the participants who registered for the Delphi study. Interviews were selected to allow the researchers use open questions with which to control the line of questioning. This allowed us to focus on the results of the intervention and subsequent additional ethnography and limit the indirect views of the interviewee (Creswell, 2003: 187).

The second ethnographic study revealed that the intervention was not routinely followedparticularly in times of great pressure (from high patient volume or where the acuity of some patients absorbed a lot of clinical time). Of the 14 patients available for study during the scheduled ethnography, only nine had visible compliance (proportion of o.64). Although all of the failures happened at times when fourhour wait pressures were most intense, four compliances were also noted during this time. Also mechanisms to monitor the indicators and resolve issues when performance was below standard were not evident: although the intervention process and its indicators were described, monitoring actual performance was not undertaken on an ongoing basis. Delayed assessment of performance was observed as common practice in the hospital. Performance against policy was seen to be managed through a periodic audit by the trust's quality department rather than by clinical staff when performance issues were occurring.

However, the interviews showed a general themed belief that the intervention was successful. This belief was based on the involvement of the clinical body who identified the performance gap and created a solution rather than responding to central or managerial directives. However, no assessment of the low compliance rate was mentioned, which was unsurprising given the lack of mechanisms to monitor the indicators.

\section{Discussion}

In evaluating the hypothesis that the enterprise culture framework in the emergency department is successful in achieving quality and efficiency 
improvements, the following key aspects of the PPP framework were identified. The intervention and its success criteria addressed local needs, which are identified as desirable factors in the effective use of performance indicators (Turner et al, 2013b). Additionally, the process was well defined and captured the expertise of a wide group of stakeholders who had a clear view of the purpose in their approach to problem solving that Womack et al (2007) consider important, although involvement from participants within the emergency department was limited for the reasons already noted. Furthermore, the effect of clinical managers guiding participants' knowledge to improve performance of a clearly defined purpose demonstrated fundamental principals of PPP framework problem-solving methods (Turner et al, 2013b).

However, sustained and complete compliance was not achieved and other key aspects of PPP were absent. All performance is the result of a process' (Womack, 2005: 3), but to achieve success people must enact the process unless a need to resolve an immediate problem occurs (Womack et al, 2007). As the case site relied on agency staff (Turner et al, 2015c), the use of people fully competent in enacting the process was compromised. Training new staff to be competent in local procedures is especially difficult where permanent, experienced staff were engaged in clinical activities to the extent that they were not available for development and training.

As the intervention's indicators were not monitored, or evidenced by the receiving $\mathrm{CDU}$, resolution of performance issues was not possible and failure to comply was only evidenced anecdotally and without closure. Some evidence of the departmental relationship concerns from our ethnographic study (Turner et al, 2015c) remained when studying the efficacy of the intervention and may have also contributed to non-compliance.

The intervention represented a localised issue for emergency care patients, but did not address the key purpose of the enterprise culture: achievement of the four-hour wait targets. Observation patients account an average of 12 out of the daily 133 attendances, but the consultants' choice of intervention was not based on quantified information, rather what felt right clinically and what would make an improved service. An intervention to provide capacity to meet demand and relieve four-hour wait pressures would have relied on resources throughout the emergency care system-not just internally to the emergency department or even the hospital, but involving ambulance providers (acute and patients transport), NHS primary care, commissioning trusts and care homes for example. The limitations of organisational restraints and internal departmental rigidity may have affected the selection.

The limitations from structural barriers to research both in terms of planning the method (potential participants and sample restrictions to evaluate the intervention efficacy), and in the live setting (the lack of availability of participants and the effect of losing a consultant from the study), presented a limitation to the study quality.

\section{Conclusions}

Through this intervention we have:

- Identified structural barriers to research in a rural DGH.

- Introduced an intervention in this live setting.

- Conducted research to evaluate the effectiveness of the intervention given the structural barriers.

We believe that our findings are useful to clinicians looking to introduce similar interventions and future researchers in comparable live settings.

Although a process capable of resolving a locally identified performance issue was implemented, problems with availability of competent staff to enact it affected its efficacy. The availability of sufficient experienced staff was also evident in the limitations of applying the protocol and validating the research. Pressure to achieve the four-hour target and inadequate volume of staff who were competent in enacting the process were responsible for the below-expected compliance.

The intervention did not widely test the enterprise culture's ability to achieve quality and efficiency aims, however, the effects from the failure to follow the PPP framework in such a 
study presents a concern. Insufficient evidence was found to support the hypothesis that the enterprise culture in the case study emergency department was successful in achieving quality and efficiency improvements. BJHCM

\section{References}

Bloor M, Sampson H, Baker S, Dahlgren K (2015) Useful but no oracle: Reflections on the use of a delphi group in a multi-methods policy research study. Qualitative Research 15(1): 57-70. doi: 10.1177/1468794113504103

Bowling A (2009) Research Methods in Health: Investigating Health and Health Services. McGrawHill International, Maidenhead

Campbell MJ, Machin D, Walters SJ (2007) Medical Statistics: A Textbook for the Health Sciences. 4th edn, Wiley, Chichester

Creswell JW (2014) Reseach Design: Qualitative, Quantitative and Mixed Methods Approaches. 4th edn, SAGE, Thousand Oaks, CA

George ML (2003) Lean Six Sigma for Service: How to Use Lean Speed and Six Sigma Quality to Improve Services and Transactions. McGraw-Hill Education, New York, NY

Green J, Thorogood N (2004) Qualitative Methods for Health Research. Sage, Thousand Oaks, CA

Hammersley M, Atkinson P (1995) Ethnography: Principles in Practice. 2nd edn. Routledge, London

Miltenburg J (2007) Level schedules for mixed-model
JIT production lines: Characteristics of the largest instances that can be solved optimally. International Journal of Production Research 45(16): 3555-77. doi: 10.1080/00207540701223394

Silvester K, Lendon R, Bevan H, Steyn R, Walley P (2004) Reducing waiting times in the NHS: Is lack of capacity the problem? Clinician in Management 12(3): 105-9

Turner P, Kane R, Jackson C (2013b) Enterprise efficiency framework: The english NHS. British Journal of Healthcare Management 19(11): 540-44

Turner P, Kane R, Jackson C (2014) The efficacy of the enterprise culture in the english NHS. British Journal of Healthcare Management 2o(3): 109-13

Turner P, Kane R, Jackson C (2015b) Evaluation of demand in a rural english hospital emergency department. British Journal of Healthcare Management 21(6): 282-8.

Turner P, Kane R, Jackson C (2015c) Ensuring capacity meets demand: A case study. British Journal of Healthcare Management 21(9): 428

Wall A, Owen BG (2003) Health policy. 2nd edn. Routledge, New York, NY

Womack JP (2005) Going Lean in Health Care. Institute for Healthcare Improvement, Cambridge, MA

Womack JP (2011) Gemba Walks. Lean Enterprises Institute, Cambridge, MA

Womack JP, Jones DT, Roos D (2007) The Machine That Changed the World: The Story of Lean ProductionToyota's Secret Weapon in the Global Car Wars That Is Now Revolutionizing World Industry. Free Press, New York, NY

\section{Why not write for us?}

\section{Interested in contributing to British Journal of Healthcare Management?}

BJHCM publishes research, review articles and commentaries on all areas relevant to healthcare management including: commissioning, technology, policy and guidance, efficiency and workforce issues.

To find information for authors, please visit our website: http://www.magonlinelibrary.com/page/bjhc/for-authors

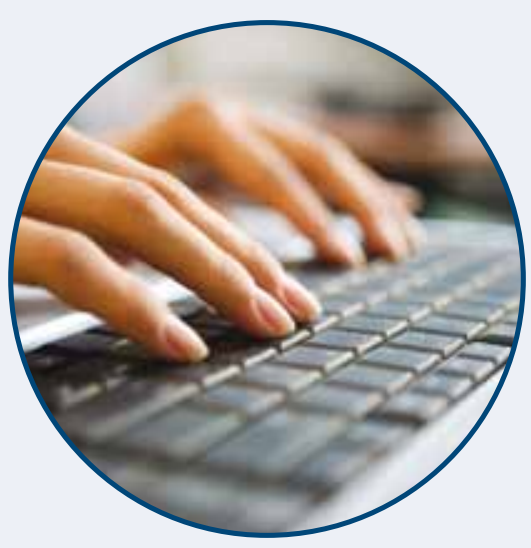

If you have an idea you would like to discuss with the editor, or have any queries about submitting an article, please write to: bjhcm@markallengroup.com 